\title{
Across the cleft
}

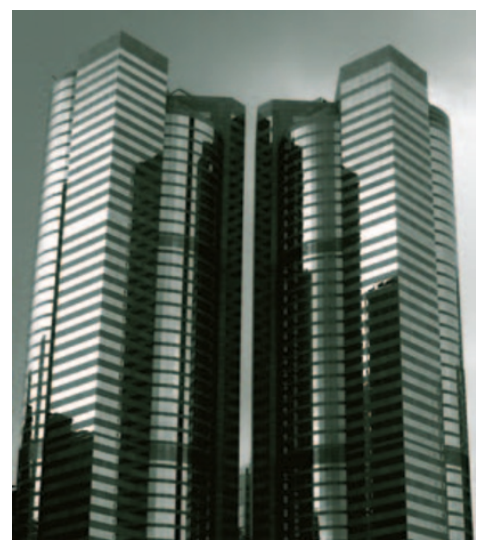

Factors released by presynaptic cells influence postsynaptic gene expression at the neuromuscular junction (NMJ) and in the CNS; however, the signalling pathways involved have not yet been identified. RavelChapuis et al. provide evidence that neural factors regulate gene expression in subsynaptic nuclei (nuclei in close proximity to the synapse) of the NMJ via chromatin acetylating and deacetylating enzymes that bind to the same transcription factor.

The less electron-dense appearance of subsynaptic versus extrasynaptic nuclei of muscles suggests that chromatin in these nuclei is less compact, which is a possible consequence of histone modifications. Neuregulin and agrin are factors that are released by motor neurons and have a pivotal role during development in the formation of NMJs, in part by upregulating the expression of acetylcholine receptors (AChRs) in muscle cells. The authors therefore investigated whether regulation of AChR gene expression in response to neural factors is governed by histone acetylation and deacetylation.

The authors carried out immunofluorescence studies on primary muscles, which demonstrated that subsynaptic, but not extrasynaptic nuclei are labelled with antibodies that recognize either hyperacetylated or phosphoacetylated histone $\mathrm{H} 3$. Moreover, intramuscular neural agrin injection showed that similar labelling could be induced in extrasynaptic nuclei located beneath the agrin-induced ectopic synapses expressing AChRs.

The activation of AChR genes in response to neural factors is known to involve two phases of gene expression: an early phase in which c-Fos and c-Jun are expressed and a late phase characterized by AChR gene expression. The authors stimulated cultured myotubes with neuregulin and showed that histone phosphorylation and acetylation also occurred in two phases that were concomitant with the activation of these two sets of genes.

Next, the authors studied protein levels of deacetylating enzymes after neuregulin treatment. They showed that neuregulin caused a decrease in the levels of the histone deacetylase HDAC1, which coincided with an increase in $\mathrm{H} 3$ acetylation in the $\varepsilon$-subunit of $\mathrm{AChR}(A C h R \varepsilon)$ promotor region. By chromatin immunoprecipitation, they also identified the histone acetyltransferase p300 as the acetylating enzyme responsible for the activation of AChR genes in response to neuregulin. Furthermore, the authors were able to establish that both HDAC1 and p300 interact with the ETS-related transcription factor GABP in a mutually exclusive fashion. GABP specifically activates transcription in response to neural factors by recruiting the histone acetylating enzyme p300 and switches to recruitment of the deacetylating enzyme HDAC1 to repress synaptic genes.

Based on these findings, the authors proposed a model in which neural factors trigger intracellular signalling cascades that activate GABP which then binds p300, resulting in the activation of gene expression. In extrasynaptic nuclei, in the absence of neural factors, GABP recruits $\mathrm{HDAC1}$, resulting in gene inactivation. It remains to be seen if this machinery is paralleled in the regulation of transcription at central synapses.

Claudia Wiedemann

ORIGINAL RESEARCH PAPER Ravel-Chapuis, A

et al. Postsynaptic chromatin is under neural control at the neuromuscular junction. EMBO J. 26, 1117-1128 (2007) 